\title{
Endothelial miR-26a regulates VEGF-Nogo-B receptor-mediated angiogenesis
}

\author{
Ha-neul Jo ${ }^{1, \#}$, Hyesoo Kang ${ }^{1, \#}$, Aram Lee ${ }^{1}$, Jihea Choi ${ }^{1}$, Woochul Chang ${ }^{2}$, Myeong-Sok Lee ${ }^{1}$ E Jongmin Kim ${ }^{1, *}$ \\ ${ }^{1}$ Division of Biological Sciences, Sookmyung Women's University, Seoul 04310, ${ }^{2}$ Department of Biology Education, College of Education, \\ Pusan National University, Busan 46241, Korea
}

\begin{abstract}
The Nogo-B receptor (NgBR) is necessary for not only Nogo-B-mediated angiogenesis but also vascular endothelial growth factor (VEGF) -induced angiogenesis. However, the molecular mechanisms underlying the regulatory role of the VEGF-NgBR axis in angiogenesis are not fully understood. Here, we report that miR-26a serves as a critical regulator of VEGF-mediated angiogenesis through directly targeting NgBR in endothelial cells (ECs). Stimulation of ECs by VEGF increased the expression of $\mathrm{NgBR}$ and decreased the expression of miR-26a. In addition, miR-26a decreased the VEGF-induced migration and proliferation of ECs. Moreover, miR-26a overexpression in ECs decreased the VEGF-induced phosphorylation of the endothelial nitric oxide synthase (eNOS) and the production of nitric oxide, which is important for angiogenesis. Overall, these data suggest that miR-26a plays a key role in VEGF-mediated angiogenesis through the modulation of eNOS activity, which is mediated by its ability to regulate $\mathrm{NgBR}$ expression by directly targeting the $\mathrm{NgBR}$ 3'-UTR. [BMB Reports 2017; 50(7): 384-389]
\end{abstract}

\section{INTRODUCTION}

Angiogenesis is the physiological process through which new blood vessels are generated from pre-existing vessels; it is not only necessary for embryologic development, normal growth, and tissue repair, but is also involved in pathological processes such as tumor growth (1). Angiogenesis is controlled by a fine balance between pro- and anti-angiogenic factors (2), with endothelial cells (ECs) being key regulators of this process. Pro-angiogenic factors, among which the vascular endothelial growth factor A (VEGF-A) is the most potent regulator of

*Corresponding author. Tel: +82-2-710-9553; Fax: +82-2-20777322; E-mail: jkim@sookmyung.ac.kr

${ }^{\#}$ These authors contributed equally to this work.

https://doi.org/10.5483/BMBRep.2017.50.7.085

Received 25 May 2017, Revised 8 June 2017, Accepted 12 June 2017

Keywords: Angiogenesis, Endothelial nitric oxide synthase, MicroRNA-26a, Nogo-B receptor, Vascular endothelial growth factor angiogenesis, exert coordinated control over multiple EC phenotypic behaviors such as migration, proliferation, differentiation, and polarity (3). During the angiogenic process, VEGF-A binds to its cognate receptor, VEGFR2 (VEGF receptor-2; also termed KDR and Flk-1), activating the elements of the angiogenic signaling cascade, such as mitogen-activated protein kinases (MAPKs) and the endothelial nitric oxide synthase (eNOS), in ECs $(1,2,4)$. Abnormal angiogenesis caused by EC dysfunction has been involved in cancer and pathological conditions associated with vessel deterioration, such as diabetic wound healing, infectious diseases, and pulmonary hypertension (5-9).

Accumulating evidence indicates an important role for Nogo-B receptor $(\mathrm{NgBR})$ signaling and the expression level of this receptor in angiogenesis (10-13). NgBR is a transmembrane receptor protein that has been identified as a Nogo-B-binding protein and is essential for the Nogo-B-mediated chemotaxis of ECs and their organization into tubes (10). In addition, NgBR is necessary for VEGF-induced angiogenesis in ECs, as the genetic knockdown of Nogo-B or $N g B R$ in a zebrafish model leads to defective intersomitic vessel formation during embryonic angiogenesis (12), with the defects being more severe in the case of $N g B R$ knockdown. More recently, it was reported that endothelial-specific $\mathrm{NgBR}$ knockout leads to early embryonic lethality in mice due to defects in vascular development and NgBR participates in this process through a Nogo-B-independent mechanism (14). These studies indicate that $\mathrm{NgBR}$ is involved in Nogo-B-independent angiogenic signaling pathways. Overall, despite the fact that the Nogo-B-NgBR axis has been found to be important in EC angiogenesis, our understanding of the role of NgBR in NogoB-independent angiogenic processes remains incomplete. Thus, the elucidation of the mechanisms underlying the regulation of angiogenic processes induced independently of Nogo-B, e.g., by the VEGF-NgBR axis, might be important. In addition, the dysregulation of $\mathrm{NgBR}$ expression leads to a wide array of diseases, including IPH, pediatric epilepsy, and cancer $(11,15-18)$, which, combined with the involvement of this receptor in the regulation of angiogenic phenotypes in ECs, indicates that the modulation of $\mathrm{NgBR}$ expression might prove useful as a new therapeutic approach for diseases caused by abnormal angiogenesis. 
This study sought to investigate the regulatory mechanisms underlying the VEGF-NgBR axis-mediated angiogenesis. We report a novel miR-26a-mediated molecular mechanism that plays a critical role in VEGF-mediated angiogenesis by regulating the expression of $\mathrm{NgBR}$.

\section{RESULTS}

\section{VEGF upregulates NgBR and reduces miR-26a expression in ECs}

To investigate the effect of VEGF on NgBR expression in ECs, human umbilical vein endothelial cells (HUVECs) were stimulated with VEGF. Both the mRNA and the protein expression of $\mathrm{NgBR}$ increased, suggesting that VEGF signaling regulates $\mathrm{NgBR}$ expression and providing clues to the molecular mechanism of VEGF-NgBR axis-mediated angiogenesis (Fig. 1A and $B$ ). The next step was the identification of the mechanisms underlying the induction of $\mathrm{NgBR}$ expression by VEGF stimulation in ECs. We first examined the possibility that microRNAs (miRNAs) acted as post-transcriptional regulators of the VEGF-induced increase in NgBR expression. The algorithms of Targetscan were used to identify miRNAs that potentially target the $3^{\prime}$-untranslated region (3'-UTR) of the $N g B R$ mRNA. The analysis predicted that miR-26a, a miRNA with highly conserved seed sequences, may bind to the 3'-UTR of NgBR at two binding sites, and could thus be a potential regulator of $\mathrm{NgBR}$ expression (Fig. 1C). We next investigated whether the expression of miR-26a is inversely
A

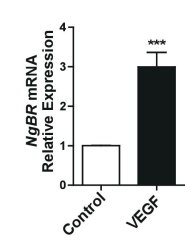

C Nogo-B receptor (NgBR)

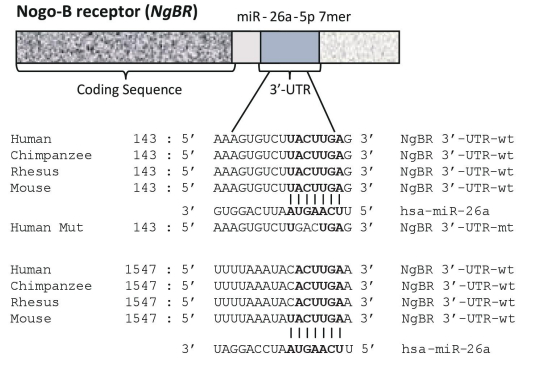

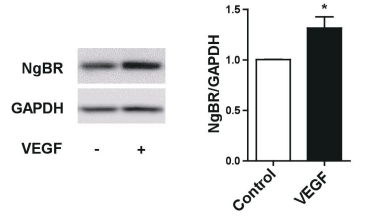

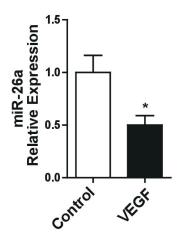

Fig. 1. VEGF upregulates NgBR and reduces miR-26a expression in HUVECs. (A) NgBR mRNA expression after treatment of HUVECs with VEGF. (B) NgBR protein expression after treatment of HUVECs with VEGF. (C) Predicted target sequences of miR-26a on the NgBR $3^{\prime}$-UTR. (D) Expression of miR-26a in response to VEGF as assessed by quantitative PCR. ${ }^{*} \mathrm{p}<0.01,{ }^{* * *} \mathrm{p}<$ 0.0001 compared to controls by unpaired two-tailed Student's $t$-test. Error bars, standard error of the mean. correlated with that of NgBR after stimulation of HUVECs with VEGF, and found that miR-26a expression was significantly lower in VEGF-treated HUVECs than in controls (Fig. 1D), suggesting a possible involvement of miR-26a in the mechanism underlying the induction of $\mathrm{NgBR}$ expression in response to VEGF.

MiR-26a regulates VEGF signaling by directly targeting NgBR To evaluate whether miR-26a is indeed involved in the regulation of $\mathrm{NgBR}$ expression, we first determined the effects of miR-26a overexpression on NgBR expression in HUVECs. Results showed that the overexpression of miR-26a in HUVECs led to a significant downregulation of both the mRNA and protein levels of $\mathrm{NgBR}$ (Fig. 2A and B, respectively). Next, to determine whether miR-26a regulates $\mathrm{NgBR}$ expression via binding directly to the $\mathrm{NgBR}$ 3'-UTR, we examined the effects of miR-26a overexpression in HEK293T cells transfected with a luciferase reporter construct containing either the wild-type or a mutant $N g B R$ 3'-UTR. Results showed a significant reduction in the luciferase activity of the NgBR 3'-UTR-wt construct, whereas the activity of the NgBR $3^{\prime}$-UTR-mt construct was not

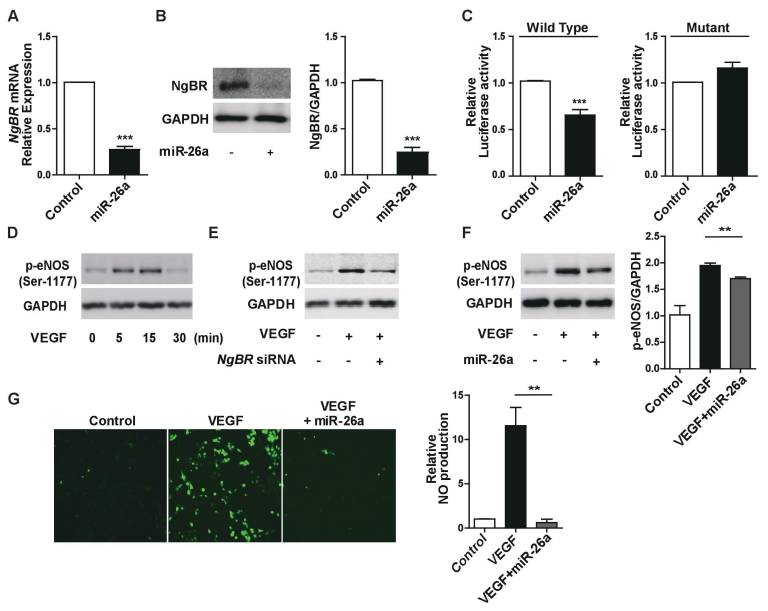

Fig. 2. MicroRNA-26a inhibits VEGF signaling by directly targeting the NgBR. (A) NgBR mRNA expression in response to the overexpression of miR-26a in HUVECs. (B) NgBR protein expression in response to the overexpression of miR-26a in HUVECs. (C) Luciferase activity after overexpression of miR-26a in HEK293T cells transfected with a reporter construct containing the wild-type (wt) and the mutant (mt) NgBR 3'-UTR. (D) Temporal changes in phosphorylation of eNOS of HUVEC cultures upon treatment with VEGF $(50 \mathrm{ng} / \mathrm{ml})$. (E) Phosphorylation of eNOS in response to VEGF (50 ng/ml) in HUVEC $N g B R$ knockdown cultures or cultures transfected with negative control siRNA. (F) Phosphorylation of eNOS in response to VEGF $(50 \mathrm{ng} / \mathrm{ml})$ in HUVECs overexpressing miR-26a or non-overexpressing control cultures. (G) Measurement of NO formation in VEGF-untreated and VEGF (50 ng/ml)-treated HUVEC cultures, as well as in VEGF-treated cultures overexpressing miR-26a. ${ }^{* *} \mathrm{p}<0.001, * * * \mathrm{p}<0.0001$ compared to controls by unpaired two-tailed Student's t-test. Error bars, standard error of the mean. 
affected (Fig. 2C). Taken together, these results suggest that miR-26a directly regulates NgBR expression in HUVECs. It was also evaluated whether the perturbation of miR-26a expression affected the phosphorylation of eNOS, which is one of the main downstream targets of VEGF-induced $\mathrm{NgBR}$ signaling in ECs and plays a key role in the regulation of angiogenesis. First, we performed a time course for the phosphorylation of eNOS in VEGF-treated HUVECs. It was found that VEGF led to maximum eNOS phosphorylation after 5-15 min and then started to decline, reaching the basal level after another 15 min, i.e., 20-30 min after stimulation (Fig. 2D). We next examined whether reducing $\mathrm{NgBR}$ expression by $\mathrm{NgBR}$ knockdown or miR-26a overexpression resulted in altered eNOS activity in VEGF-stimulated HUVECs and found that both manipulations reduced VEGF-induced eNOS phosphorylation (Fig. 2E and F). Given that eNOS catalyzes the production of $\mathrm{NO}$, a molecule involved in angiogenesis through its role as an important downstream mediator of angiogenic factors such as VEGF, we also assessed NO levels by measuring DAF fluorescence. VEGF significantly increased DAF fluorescence, an effect that was completely abrogated by concurrent miR-26a overexpression (Fig. 2G). Overall, these findings suggest an important role for the miR-26a-NgBReNOS signaling pathway in VEGF-induced angiogenesis.

NgBR inhibition attenuates VEGF-induced migration in ECs Given the angiogenic properties of VEGF in ECs and its ability to upregulate $\mathrm{NgBR}$ expression, migration assays were utilized
A
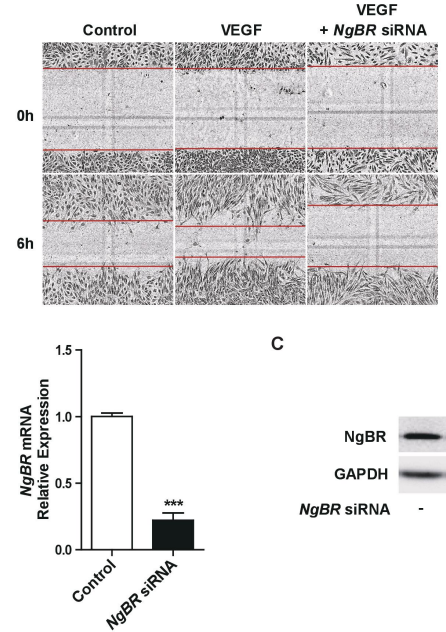

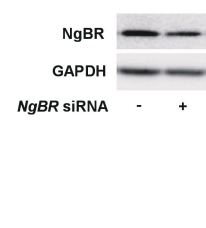

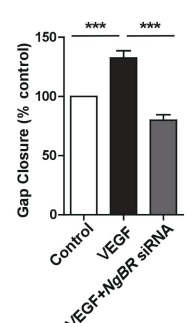

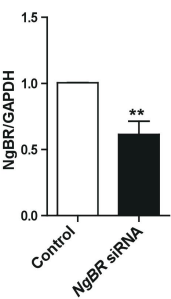

Fig. 3. NGBR knockdown abrogates the VEGF-induced endothelial migration. (A) Cell migration assays after VEGF (50 ng/ml) treatment of HUVEC cultures transfected with $N g B R$ siRNA or negative control siRNA. (B) NgBR mRNA expression in HUVEC cultures after $\mathrm{NgBR}$ knockdown. (C) $\mathrm{NgBR}$ protein expression in HUVECs after NgBR knockdown. ${ }^{* *}$ p $<0.001, * * * p<0.0001$ vs. controls, calculated by unpaired two-tailed Student's $t$-tests. Error bars, standard error of the mean. to investigate the role of $\mathrm{NgBR}$ in VEGF-induced angiogenesis. As shown in Fig. 3A, VEGF significantly induced HUVEC migration, an effect that was completely abrogated when $\mathrm{NgBR}$ was knocked down. The effectiveness of the knockdown was validated by determining the mRNA and protein levels of $\mathrm{NgBR}$. Results showed significant reductions in both of them (roughly $80 \%$ and $40 \%$, respectively; Fig. $3 \mathrm{~B}$ and C). These findings indicate a key role for $\mathrm{NgBR}$ signaling in the regulation of VEGF-mediated angiogenesis.

\section{MiR-26a impairs pro-angiogenic phenotypes in ECs}

To characterize the role of endothelial miR-26a in angiogenesis, we first assessed the effects of miR-26a overexpression on the proliferation and the migratory ability of HUVECs cultured in complete endothelial cell medium and found that it significantly reduced both properties (Fig. 4A and B), indicating that miR-26a exerts a potential anti-angiogenic function in HUVECs stimulated by complete media. The finding that miR-26a directly regulates $\mathrm{NgBR}$ expression led us to investigate the effects of miR-26a overexpression on VEGF-induced angiogenesis by performing proliferation,

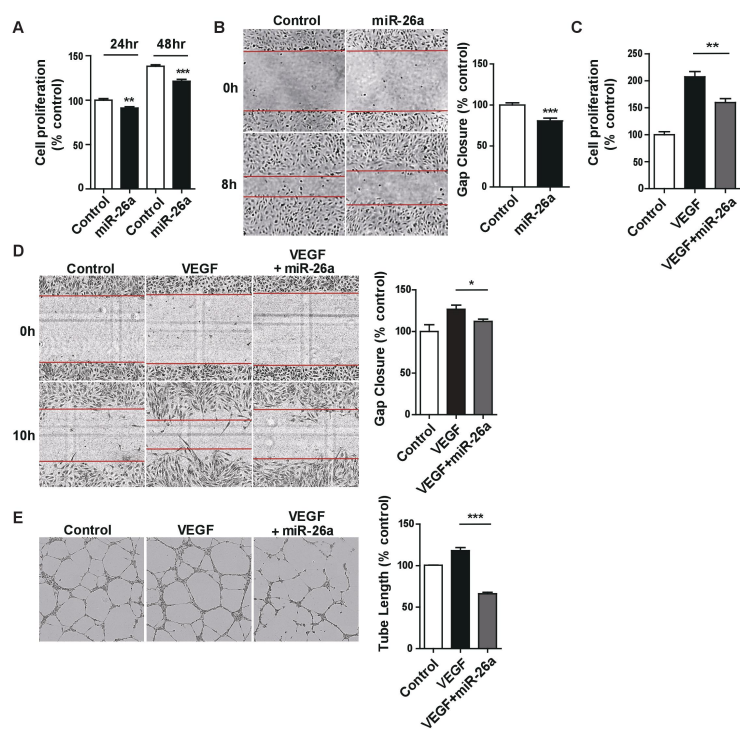

Fig. 4. MicroRNA-26a impairs pro-angiogenic phenotypes in HUVECs. (A) Relative HUVEC viability $24 \mathrm{~h}$ and $48 \mathrm{~h}$ after the overexpression of miR-26a. (B) HUVEC migratory ability $8 \mathrm{~h}$ after the overexpression of miR-26a. (C) Relative viability in response to VEGF $(50 \mathrm{ng} / \mathrm{ml})$ in HUVEC cultures overexpressing miR-26a or non-overexpressing control cultures. (D) Cell migration in response to VEGF $(50 \mathrm{ng} / \mathrm{ml})$ in HUVEC cultures overexpressing miR-26a or non-overexpressing control cultures. (E) Tube formation in response to VEGF $(50 \mathrm{ng} / \mathrm{ml})$ by HUVECs overexpressing miR-26a overexpression or non-overexpressing control cultures. ${ }^{*} \mathrm{p}<0.01, * * \mathrm{p}<0.001,{ }^{* * *} \mathrm{p}<0.0001$ compared to controls by unpaired two-tailed Student's t-test. Error bars, standard error of the mean. 
migration, and tube formation assays in HUVECs. As expected, the overexpression of miR-26a significantly reduced VEGFinduced HUVEC proliferation, migration, and tube formation (Fig. 4C-E, respectively). Taken together, the results of this study suggest an important role for the miR-26a-NgBR signaling pathway in VEGF-induced angiogenesis in ECs.

\section{DISCUSSION}

Although NgBR-mediated angiogenesis has drawn much attention, only a few studies have examined the role of $\mathrm{NgBR}$ in this process, in particular with regard to the underlying molecular mechanism regulating $\mathrm{NgBR}$ expression during angiogenesis and the mechanistic role of NgBR in Nogo-Bindependent angiogenesis, such as the one mediated by the VEGF-NgBR axis. This study demonstrated the essential role of miR-26a in regulating VEGF-induced NgBR expression and, by extension, the angiogenic effects of this protein. These findings can be summarized into four major conclusions: (i) $\mathrm{NgBR}$ and miR-26a expression levels are reversely correlated in VEGF-stimulated HUVECs. (ii) NgBR is a direct target of miR-26a. (iii) miR-26a inhibits VEGF-induced angiogenic phenotypes in HUVECs. (iv) miR-26a inhibits VEGF-induced eNOS phosphorylation and NO production.

Previous studies have demonstrated that NgBR plays an important role in various physiological and pathological processes, such as angiogenesis, development, and tumor growth (11-17). Most of these studies focused on investigating the effects of NgBR overexpression or knockdown and simply determining the $\mathrm{NgBR}$ expression levels in vitro or in vivo. Given that the control of NgBR expression is critical to the regulation of angiogenesis, the molecular mechanism underlying this regulation in ECs is important for assessing the angiogenic process. In recent years, much attention has been paid to the role of miRNAs in angiogenesis, particularly VEGF-targeting or VEGF-responsive miRNAs. For example, miR-16, the miR-424/503 cluster, miR-126, and miR-497 are anti-angiogenic miRNAs that directly target the VEGF mRNA in ECs and cancer cells (19-22), whereas the miR-17-92 cluster, miR-16, and miR-424 respond to VEGF stimulation and modulate EC angiogenic responses $(19,23)$.

MiR-26a is highly expressed in ECs (24) and is known to be a key regulator of EC functions such as angiogenesis and apoptosis $(18,25,26)$. Previous studies have shown that the ectopic expression of miR-26a leads to the inhibition of angiogenesis, whereas the administration of miR-26a inhibitors induces the angiogenic properties of ECs by activating BMP/SMAD1 signaling $(26,27)$. Furthermore, miR-26a exerts anti-apoptotic activity by directly targeting TRPC6 in ECs (25). Taken together, all these evidences suggest that the modulation of miR-26a may represent a new therapeutic option for vascular diseases. However, the roles of endothelial miR-26a in VEGF-mediated angiogenesis had not been determined. This study is the first to reveal the role of miR-26a in VEGF-induced angiogenesis and report on the molecular mechanisms involved in the regulation of $\mathrm{NgBR}$ expression. The current findings demonstrate that miR-26a reduces the expression of $\mathrm{NgBR}$ by directly targets $\mathrm{NgBR}$ reducing its expression, and the downregulation of miR-26a is the mechanism through which VEGF upregulates NgBR. Restoration of miR-26a expression results in NgBR downregulation, which in turn leads to the inhibition of the VEGF-induced angiogenic phenotypes including proliferation, migration, and the formation of capillary-like structures. In addition, this study demonstrated for the first time that miR-26a can regulate eNOS activity and NO production in VEGF-treated HUVECs. Since, as was also shown in this study, miR-26a targets NgBR expression, it may act as a key regulator of VEGF-induced NO production and, by extension, its angiogenic properties.

Overall, our research provides novel mechanistic insights into the angiogenesis signaling processes taking place in VEGF-stimulated endothelium. Specifically, it establishes a novel regulatory mechanism of VEGF-mediated angiogenesis, namely the ability of miR-26a to downregulate endothelial NgBR expression by directly targeting the $3^{\prime}-\mathrm{UTR}$ of the $\mathrm{NgBR}$ mRNA. Moreover, it proposes that the modulation of eNOS phosphorylation constitutes the downstream signaling mechanism regulated by the miR-26a-NgBR axis.

\section{MATERIALS AND METHODS}

\section{Cell culture}

HUVECs were grown in endothelial cell growth medium-2 (EGM-2; Lonza, Basel, Switzerland) supplemented with 1\% penicillin-streptomycin (WelGENE, Daegu, Korea) at $37^{\circ} \mathrm{C}$ in a $5 \% \mathrm{CO}_{2}$ incubator. HEK293T cells were cultured in HyClone Dulbecco's modified Eagle's medium (DMEM; GE Healthcare Life Sciences, Marlborough, IL, USA) containing 10\% fetal bovine serum (FBS; Gibco, Gaithersburg, MD, USA) and 1\% penicillin-streptomycin. Both cell types were cultured to $70-80 \%$ confluency before each experiment.

\section{Transfection}

Lipofectamine RNAimax and Lipofectamine 2000, both obtained from Invitrogen (Carlsbad, CA, USA), were used for miRNA and siRNA transfection following the manufacturer's instructions. The control miRNA and the miR-26a mimics used in in vitro transfections were also provided by Invitrogen. NGBR siRNAs (forward: 5'-GGAAAUACAUAGACCUACA-3', reverse: 5'-UGUAGGUCUAUGUAUUUCC-3') were synthesized from Genolution (Seoul, Korea). Stealth RNAi (ST Pharm, Ansan, Korea) was used as the negative control siRNA.

\section{RNA extraction and quantitative real-time PCR}

Total RNA was isolated using the miRNeasy RNA isolation kit (Qiagen, Hilden, Germany). Purified RNA was reversetranscribed using the TaqMan miRNA Reverse Transcription Kit (Applied Biosystems, Carlsbad, CA, USA). For microRNA 
amplification, the qPCR Universal Master Mix II, no UNG (Applied Biosystems) was used and miR-26a was detected with Taqman probes. RNU6B and 18S RNA were used as internal controls for the quantification of miR-26a and the $\mathrm{NgBR}$ $m R N A$, respectively. The primers for the PCR amplification of $N g B R$ mRNA and 18S rRNA amplification were the following: $\mathrm{NgBR}$ forward, 5'-AGAGCTGCTCAGGACTTTTGCC-3'; NgBR reverse, 5'-TGCTGTCCACAGGACCGAACTT-3'; 185 forward, 5'-ACCCGTTGAACCCCATTCGTGA-3'; 185 reverse, 5'-GCCT CACTAAACCATCCAATCGG-3'.

\section{Western blotting}

HUVECs were lysed with RIPA buffer (GenDEPOT, Barker, TX, USA) containing protease and phosphatase inhibitor cocktail (Roche, Basel, Switzerland). Protein quantification was performed using the Pierce BCA Protein Assay kit (Thermo Fisher Scientific, Waltham, MA, USA) and equal protein concentrations were boiled, loaded into SDS-polyacrylamide gels, electrophoresed, and transferred to polyvinyl difluoride membranes (Merck Millipore, Billerica, MA, USA). Blots were treated with primary antibodies against $\operatorname{NgBR} \quad(1: 2,000$; Abcam, Cambridge, MA, USA), phospho-eNOS (Ser-1177) (1:2,000; \#612392; BD Bioscience, Franklin Lakes, NJ, USA), and GAPDH (1:5,000; \#2118; Cell Signaling Technology). For immunodetection and development, HRP-conjugated secondary antibodies (1:3,000, Cell Signaling Technology) and an enhanced chemiluminescence detection system (Thermo Fisher Scientific) were used.

\section{Luciferase reporter assay}

The human NgBR 3'-UTR (2,144 bp), including the predicted binding sites of the two miR-26a seed sequences, was cloned between the Not1 and Xhol sites of the psiCHECK2 vector (Promega, Fitchburg, WI, USA), upstream of the Renilla luciferase coding region. We also created a construct containing a mutated NgBR $3^{\prime}-U T R$ in which the first miR-26abinding site had been altered (TTACTTG to TTGACTG) using the Muta-DirectTM Site-Directed Mutagenesis Kit (iNtRON Biotechnology, Seongnam, Korea). HEK293T cells were transfected with a luciferase reporter construct (containing either the wild-type or the mutant $N g B R$ 3'-UTR) and miRNA (either the miR-26a mimic or negative control miRNA) using lipofectamine 2000. Cells were lysed after a 48-h incubation period and luciferase activity was measured using the Dual-Luciferase Reporter Assay System (Promega).

\section{Tube formation assay}

HUVECs were seeded on 6-well plates and transfected with miR-26a mimics using RNAimax (Invitrogen). After $24 \mathrm{~h}$, cells were trypsinized, counted, and seeded at $1.5 \times 10^{4}$ cells/well in 48-well plates pre-coated with phenol-red free, growth factor reduced (GFR) Matrigel Matrix (BD Bioscience). For treatments of VEGF (R\&D Systems, Minneapolis, MN, USA), it was suspended in EBM-2 medium and used at the concentration of $50 \mathrm{ng} / \mathrm{ml}$. The formation of tube-like structures was observed every $2 \mathrm{~h}$ under an optical microscope at $40 \times$ magnification and quantified with the Image J software (Open Access, Public Domain).

\section{Proliferation assay}

HUVECs were seeded at a density of $5 \times 10^{3}$ cells/well in 96-well plates. Following overnight incubation, cells were transfected with $24 \mathrm{nM}$ of miR-26a mimic. After $24 \mathrm{~h}$, the EGM-2 complete medium was removed and replaced with starvation medium. After another $12 \mathrm{~h}$, VEGF $(50 \mathrm{ng} / \mathrm{ml})$ was added to the medium. Following incubation for another $24 \mathrm{~h}$, $20 \mu \mathrm{l}$ of the WST-1 reagent was added to each well and incubated for $1 \mathrm{~h}$. The absorbance was measured by a microplate reader at $450 \mathrm{~nm}$ with a background reference wavelength of $620 \mathrm{~nm}$. For assessing the effects of treatment with miR-26a only, the WST-1 reagent was added 24 or $48 \mathrm{~h}$ after transfection.

\section{Migration assay}

HUVECs were seeded at a density of $2 \times 10^{5}$ cells/well in 12-well plates. After $24 \mathrm{~h}$, cells were transfected with miR-26a mimic at $24 \mathrm{nM}$ or $\mathrm{NgBR}$ siRNA at $36 \mathrm{nM}$. Cells were scratched with a P-200 pipette tip and incubated in starvation medium containing $1 \%$ FBS. VEGF (50 ng/ml) was added after scratching. Cells were observed under an optical microscope at $\times 40$ magnification and measured using Imagej.

\section{Nitric oxide measurement}

HUVECs were seeded in 6-well plates and transfected with miR-26a mimic at $24 \mathrm{nM}$. After $16 \mathrm{~h}$, media were replaced by serum-free medium. VEGF $(50 \mathrm{ng} / \mathrm{ml})$ was added to the starved HUVECs for 15 minutes. Carefully (to avoid detachment), cells were stained with DAF-FM diacetate at $5 \mu \mathrm{M}$ under dark at $37^{\circ} \mathrm{C}$. After the removal of excess probe, the relative levels of intracellular $\mathrm{NO}$ were determined by measuring their fluorescence intensity under a fluorescence microscope.

\section{ACKNOWLEDGEMENTS}

Ha-neul Jo was provided support by the Sookmyung Women's University BK21 Plus Scholarship. I would like to thank Dr. Danielle L. McLean for her critical reading of the manuscript and thoughtful discussions. This study was supported by grants from the National Research Foundation of Korea (Basic Science Research Program NRF-2016R1A5A1011974 and NRF-2016R1C1B2006591 to J.K) and the Korea Health Technology R\&D Project through the Korea Health Industry Development Institute (KHIDI), funded by the Ministry of Health \& Welfare, Republic of Korea (HI13C1372 to J.K).

\section{CONFLICTS OF INTEREST}

The authors have no conflicting financial interests. 


\section{REFERENCES}

1. Carmeliet $P$ (2005) Angiogenesis in life, disease and medicine. Nature 438, 932-936

2. Iruela-Arispe ML and Dvorak HF (1997) Angiogenesis: a dynamic balance of stimulators and inhibitors. Thromb Haemost 78, 672-677

3. Herbert SP and Stainier DY (2011) Molecular control of endothelial cell behaviour during blood vessel morphogenesis. Nat Rev Mol Cell Biol 12, 551-564

4. Kim J, Park J, Choi S et al (2008) X-linked inhibitor of apoptosis protein is an important regulator of vascular endothelial growth factor-dependent bovine aortic endothelial cell survival. Circ Res 102, 896-904

5. Carmeliet $P$ and Jain RK (2000) Angiogenesis in cancer and other diseases. Nature 407, 249-257

6. Kang H, Park Y, Lee A et al (2017) Negative regulation of NOD1 mediated angiogenesis by PPARgamma-regulated miR-125a. Biochem Biophys Res Commun 482, 28-34

7. Kim J, Kang Y, Kojima $Y$ et al (2013) An endothelial apelin-FGF link mediated by miR-424 and miR-503 is disrupted in pulmonary arterial hypertension. Nat Med $19,74-82$

8. Kim J, Hwangbo C, Hu X et al (2015) Restoration of impaired endothelial myocyte enhancer factor 2 function rescues pulmonary arterial hypertension. Circulation 131, 190-199

9. Kim J (2014) Apelin-APJ signaling: a potential therapeutic target for pulmonary arterial hypertension. Mol Cells 37, 196-201

10. Miao RQ, Gao Y, Harrison KD et al (2006) Identification of a receptor necessary for Nogo-B stimulated chemotaxis and morphogenesis of endothelial cells. Proc Natl Acad Sci U S A 103, 10997-11002

11. Teng RJ, Rana U, Afolayan AJ et al (2014) Nogo-B receptor modulates angiogenesis response of pulmonary artery endothelial cells through eNOS coupling. Am J Respir Cell Mol Biol 51, 169-177

12. Zhao B, Chun C, Liu Z et al (2010) Nogo-B receptor is essential for angiogenesis in zebrafish via Akt pathway. Blood 116, 5423-5433

13. Rana U, Liu Z, Kumar SN et al (2016) Nogo-B receptor deficiency causes cerebral vasculature defects during embryonic development in mice. Dev Biol 410, 190-201

14. Park EJ, Grabinska KA, Guan Z and Sessa WC (2016) $\mathrm{NgBR}$ is essential for endothelial cell glycosylation and vascular development. EMBO Rep 17, 167-177

15. Pula B, Olbromski M, Owczarek T et al (2014) Nogo-B receptor expression correlates negatively with malignancy grade and ki-67 antigen expression in invasive ductal breast carcinoma. Anticancer Res 34, 4819-4828

16. Pula B, Werynska B, Olbromski M et al (2014) Expression of Nogo isoforms and Nogo-B receptor (NgBR) in non-small cell lung carcinomas. Anticancer Res 34, 4059-4068

17. Wang B, Zhao B, North P et al (2013) Expression of NgBR is highly associated with estrogen receptor alpha and survivin in breast cancer. PLoS One 8, e78083

18. Szafranski P, Von Allmen GK, Graham BH et al (2015) $6 q 22.1$ microdeletion and susceptibility to pediatric epilepsy. Eur J Hum Genet 23, 173-179

19. Chamorro-Jorganes A, Araldi E, Penalva LO et al (2011) MicroRNA-16 and microRNA-424 regulate cell-autonomous angiogenic functions in endothelial cells via targeting vascular endothelial growth factor receptor-2 and fibroblast growth factor receptor-1. Arterioscler Thromb Vasc Biol 31, 2595-2606

20. Zhou B, Ma R, Si W et al (2013) MicroRNA-503 targets FGF2 and VEGFA and inhibits tumor angiogenesis and growth. Cancer Lett 333, 159-169

21. Zhang Y, Wang X, Xu B et al (2013) Epigenetic silencing of miR-126 contributes to tumor invasion and angiogenesis in colorectal cancer. Oncol Rep 30, 1976-1984

22. Wang W, Ren F, Wu Q et al (2014) MicroRNA-497 suppresses angiogenesis by targeting vascular endothelial growth factor $A$ through the PI3KJAKT and MAPK/ERK pathways in ovarian cancer. Oncol Rep 32, 2127-2133

23. Chamorro-Jorganes A, Lee MY, Araldi E et al (2016) VEGF-Induced Expression of miR-17-92 Cluster in Endothelial Cells Is Mediated by ERK/ELK1 Activation and Regulates Angiogenesis. Circ Res 118, 38-47

24. Harris TA, Yamakuchi $M$, Ferlito $M$ et al (2008) MicroRNA-126 regulates endothelial expression of vascular cell adhesion molecule 1. Proc Natl Acad Sci U S A 105, 1516-1521

25. Zhang $Y$, Qin W, Zhang L et al (2015) MicroRNA-26a prevents endothelial cell apoptosis by directly targeting TRPC6 in the setting of atherosclerosis. Sci Rep 5, 9401

26. Icli B, Nabzdyk CS, Lujan-Hernandez J et al (2016) Regulation of impaired angiogenesis in diabetic dermal wound healing by microRNA-26a. J Mol Cell Cardiol 91, 151-159

27. Icli B, Wara AK, Moslehi J et al (2013) MicroRNA-26a regulates pathological and physiological angiogenesis by targeting BMP/SMAD1 signaling. Circ Res 113, 12311241 